\title{
Tangence
}

\section{Chez nous d'Adjutor Rivard : esthétique et fortune littéraire}

\section{Marie-Andrée Beaudet}

Numéro 40, mai 1993

Régionalismes littéraires de la francophonie

URI : https://id.erudit.org/iderudit/025764ar

DOI : https://doi.org/10.7202/025764ar

Aller au sommaire du numéro

Éditeur(s)

Tangence

ISSN

0226-9554 (imprimé)

1710-0305 (numérique)

Découvrir la revue

Citer cet article

Beaudet, M.-A. (1993). Chez nous d'Adjutor Rivard : esthétique et fortune

littéraire. Tangence, (40), 28-38. https://doi.org/10.7202/025764ar d'utilisation que vous pouvez consulter en ligne.

https://apropos.erudit.org/fr/usagers/politique-dutilisation/ 


\title{
Chez nous d'Adjutor Rivard: esthétique et fortune littéraire
}

\author{
Marie-Andrée Beaudet*
}

On peut tenter de définir l'esthétique régionaliste, comme toute esthétique d'ailleurs, à partir des textes critiques qui ont contribué à la faire naître et à la propager, mais on peut aussi chercher à la faire émerger des œuvres elles-mêmes en analysant les particularités formelles de leur composition. Outre qu'elle permet de mettre au jour la distance qui sépare souvent la théorie de la pratique, la seconde approche permet aussi de voir comment le travail de fictionnalisation, instance première des médiations entre le social et le littéraire, joue et déjoue les volontés et les déclarations d'intention en se confrontant, dans la matière même de la langue, à l'univers des contraintes et des possibles contenus dans le champ littéraire d'une époque.

Le double caractère, a la fois critique et "romanesque", de l'œuvre d'Adjutor Rivard, l'un des principaux animateurs du mouvement régionaliste au Québec, offre à cet égard un exemple saisissant des failles et des contradictions que la fiction peut instaurer dans la logique bétonnée du discours critique. Rivard est, en fait, l'auteur d'une seule œuvre de fiction mais édifiée en deux temps. Le premier volet Cbez nous ${ }^{1}$ paraît à Québec en 1914 et le second, Chez nous, chez nos gens ${ }^{2}$, en 1919. Â l'exception de deux textes, "Un poète illettré * et "Les écumeurs de tonnes *, qui se voient remplacés par "Il n'est plus du temps", le nouveau recueil reprend les textes déjà publiés en leur adjoignant dix courts textes de la même coulée.

L'intérêt du recueil de 1914, Chez nous, tient à son caractère exemplaire $^{3}$. En effet, dans sa foulée paraîtront, dans la période

- Marie-Andrée Beaudet est professeure à l'Université Laval. Elle a publié Langue et littérature au Québec, 1895-1914 (1991) et Charles ab der Halden. Portrait d'un inconnu (1992).

1 Chez nous, Québec, Laction sociale catholique, 1914, 145 p.

2 Chez nous, chez nos gens, Québec, Éditions de l'Action sociale catholique, 1919, 257 p.

3 Le titre de l'un des récits du recueil, "L'heure des vaches", sera utilisé par les opposants au régionalisme comme texte-repoussoir. 
de l'entre-deux-guerres, toute une myriade de petits récits paysans participant de la même esthétique. Signalons quelques titres: Les rapaillages de l'abbé Lionel Groulx (1916), Premières semailles de Georges Bouchard (1917), Les choses qui s'en vont du frère Gilles (1918), Aux sources canadiennes de Georges-Émile Marquis (1918), Récits laurentiens du frère Marie-Victorin (1919), Récits et légendes de Blanche Lamontagne-Beauregard (1924), Vieilles choses, vieilles gens de Georges Bouchard (1926) ${ }^{4}$. À ces titres, il faut encore ajouter les recueils de textes publiés à l'occasion des grands concours littéraires organisés par la Société Saint-Jean-Baptiste de Montréal: La croix du chemin (1916), la convée (1917), Fleurs de lys (1918), Au pays de l'érable (1919).

Si le recueil d'Adjutor Rivard paraît être celui qui a joui de la plus grande faveur auprès du public et de la critique, la paternité du genre doit cependant être attribuée à son fidèle compagnon d'armes, l'abbé Camille Roy. Dès 1905, et non en 1913 comme plusieurs commentateurs l'affirment, Camille Roy offre aux lecteurs du quotidien Le soleil un court récit à résonance poétique qui s'impose comme le modèle de la prose régionaliste. La précision chronologique est importante car elle permet de situer la contribution littéraire de Camille Roy dans le sillage immédiat de son célèbre manifeste datant de 1904 en faveur de la "Nationalisation de la littérature canadienne. 5 . "Le vieux hangar " 6 paraît d'abord sous le pseudonyme de Benj. DesAnges, puis devant l'intérêt que le texte suscite, son auteur le reprend sous son véritable nom dans Propos canadiens ${ }^{7}$ (1912) et dans Propos rustiques ${ }^{8}$

4 Les Rapaillages (vieilles choses, vieilles gens), Montréal, Le Devoir, 1916, 159p.; Bibliothèque de l'Action française, 1919, 139 p.; Premières semailles, Québec, Imprimerie de l'Action sociale limitee, 1917, 96 p.; Les choses qui s'en vont. Causettes canadiennes, Montréal, Editions de la Tempérance, 1918,64 p. (édition revue et augmentée, 1918, 186 p.); Aux sources canadiennes, Québec, [s.é.], 1918, XVI-178 p.; Récits laurentiens, Montréal, les Frères des Écoles chrétiennes, 1919, 207 p.; Récits et légendes, Montréal, Librairie Beauchemin, 1924, 124 p.; Vieilles choses, vieilles gens, silbouettes campagnardes, Montréal, Beauchemin, 1926, $189 \mathrm{p}$.

5 Conférence donnée en 1905 et publiée dans les Essais sur la littérature canadienne, Québec, Librairie Garneau, 1907, 376 p. [p. 187 à 201].

6 . Le vieux hangar, Le soleil, 28 octobre 1905, p. 7 (Propos du samedi).

7 Propos canadiens, Québec, Imprimerie de l'Action sociale, 1912, VIII326 p.

8 Propos rustiques, Québec, Librairie Beauchemin, coll. *Dollard., 1924, 119 p. 
(1924). Le récit met en scène les plaintes et les tourments d'un vieux bâtiment qui dans "la lumière tiède et blonde de nos soleils d'automne " - la phrase revient comme un leitmotiv tout au long du texte - se souvient des heures heureuses où ses murs abritaient les travaux et les réjouissances de ses "doux maîtres". Le motif, que l'on trouvera d'ailleurs au cœur de toute la production régionaliste, est donné par un exergue empruntée à Sully Prudhomme:

Je n'aime pas les maisons neuves:

Leur visage est indifferent.

Les anciennes ont l'air de veuves

Qui se souviennent en pleurant

Contrairement à son devancier, Adjutor Rivard n'appuyera aucun des courts récits contenus dans son recueil Cbez nous ${ }^{9}$ de références extérieures. L'homogénéité de l'univers à dépeindre semble exclure toute possibilité d'emprunt. Un homme parle aux siens des choses de chez eux. C'est le sens de la dédicace:

À mes enfants,

Antoine et Georgine,

J'ai écrit pour vous ces pages, toutes simples et sans autres ornements que les mots de notre vieux parler, mais toutes vraies et qui peut-être assureront dans votre mémoire la survivance de quelques souvenirs anciens.

Puissent-elles vous faire aimer plus encore les gens et les choses de chez nous!

L'aspect testamentaire du projet n'autorise aucune voix étrangère à venir troubler la quiétude de l'espace des réminiscences privées. S'il adopte la même posture nostalgique que Camille Roy et puise à la même thématique de la disparition et de la perte, Rivard innove cependant sur le plan des stratégies lexicales.

\section{Une trajectoire exemplaire}

Adjutor Rivard s'intéressa très tôt aux questions de langue. Rappelons qu'il naît en 1868 à Saint-Grégoire-de-Nicolet mais que cette même année sa famille va s'établir à Québec, dans la rue

9 Il faut peut-être souligner que le titre du recueil renvoie au titre d'un poème d'Achille Milien, poète nivernais, que le Bulletin du parler français avait publié dans sa livraison de septembre 1904 (p. 208-209). 
des Casernes. Contrairement à ce que son œuvre porte à croire, Rivard eut une enfance urbaine, dans un milieu bourgeois et cultivé. Son père était notaire et, selon l'un de ses biographes, il " ne négligea rien pour inculquer à son fils une solide formation tant religieuse et morale qu'intellectuelle"10. Le jeune Rivard fait ses études classiques au Petit séminaire de Québec et étudie le droit à l'Université Laval. Reçu avocat en 1891 puis nommé juge de la Cour d'appel en 1921, sa carrière professionnelle est inscrite sous le signe du succès, des honneurs et de l'ascension sociale. L'adoption des thèses régionalistes ne saurait correspondre chez lui à une tentative de transcender une expérience personnelle ou familiale de l'échec, comme l'a démontré Rémy Ponton dans ses analyses de trajectoire des écrivains régionalistes français ayant publié entre 1860 et $1914^{11}$. Les motifs de son engagement doivent davantage être cherchés du côté de sa lecture de l'expérience collective et nationale.

Tout en poursuivant ses activités juridiques, Adjutor Rivard devient, en 1897, titulaire de la chaire de diction à l'Université Laval. Il publie en 1898 L'art de dire. Traité de lecture et de récitation $^{12}$ et en 1901 son Manuel de la parole ${ }^{13}$ qui connaîtra plusieurs rééditions et dans lequel, selon l'abbé Émile Chartier, "il pourchassait les malformations que notre paresse, notre ignorance ou notre indifférence faisaient subir à notre langage courant " ${ }^{14}$. Dans l'avertissement qui ouvre le Manuel, l'auteur définit son credo esthétique:

L'idéal ne se crée pas de toutes pièces dans le cerveau de l'homme; l'artiste le découvre par l'interprétation de la nature, en remontant de l'effet à la cause, de la fin au principe, de la matière à la forme. Et puisque la création est le chef-d'œuvre des chefs-d'œuvre d'art, et puisque Dieu en est l'auteur, l'artiste qui veut manifester au dehors les conceptions de son âme, ne peut mieux faire que de $s$ 'inscrire au nombre des disciples du

10 Lucien Papillon, "Adjutor Rivard ", thèse de l'École des Bibliothécaires de l'Université de Montréal, 1949, 77 f. [f. 1].

11 Rémy Ponton, *Traditions littéraires et tradition scolaire. L'exemple des manuels de lecture de l'école primaire française: quelques hypothèses de travail ", Lendemains, 1984, $\mathrm{n}^{\circ} 36$, p. 53-63.

12 Adjutor Rivard, L'art de dire. Traité de lecture et de récitation, Québec, H. Chasse, 1898, 277 p.

13 Adjutor Rivard, Manuel de la parole, Québec, J.-P. Garneau, 1901, 303 p.

14 Emile Chartier, Etude d'auteur canadien. Adjutor Rivard *, Lectures, octobre 1961, p. 68. 
32

Créateur, d'aller à sa divine école, et de s'instruire au spectacle de la nature, afin d'en imiter les perfections. ${ }^{15}$

\section{Une esthétique du labeur}

Ces notions cardinales de formation, d'imitation et de patient labeur, associées à la création artistique, se trouvent illustrées $a$ contrario dans l'un des récits du recueil Chez nous: "Un poète illettré., Rivard met en scène un "brave paysan ", Pierre-Paul, qui bien que "Né poète * est condamné par son manque d'instruction à ne produire que de mauvais vers:

Pierre-Paul, Pierre-Paul! J'ai mangé nos légumes et j'ai lu vos vers. Hélas! vos vers ne valent pas vos légumes. Et vos gretons, ô Pierre-Paul, vos gretons sont vos meilleurs poèmes!... ${ }^{16}$

S'érigeant en juge, le narrateur, après avoir cité quelques strophes de l'infortuné poète, raille la pauvreté de sa langue natale:

Eh quoi! vous trouvez que victoire ne rime pas avec altière?...

Vous ne savez pas que Pierre-Paul est du grand siècle; bon canadien, il prononce victouère, et c'est pourquoi il fait d'ailleurs rimer exploits avec guérets. ${ }^{17}$

Tout le malheur de Pierre-Paul vient de ce qu'il n'a pas fait d'études supérieures. Hors d'elles, point de réussite littéraire! Rivard partage en cela les convictions exprimées par l'abbé Camille Roy dans son Manuel de littérature canadienne ${ }^{18}$, comme l'a démontré Lucie Robert:

Très tôt le manuel établit que le bon écrivain est celui qui a fait de "fortes études classiques*. Tant pis pour les cancres et pour les pauvres. Camille Roy illustre bien ce qu'il veut dire:

- François-Xavier Garneau n'a pu fréquenter que l'école primaire. Il dut suppléer par des études personnelles à la formation qui lui a manqué. [... En conséquence] l'historien [n'a] pas toujours apprécié ou jugé comme il le fallait les questions relatives au rôle du clergé dans notre histoire.

15 Adjutor Rivard, op. cit.

16 . Un poète illettrén, p. 112.

17 Ibid., p. 114.

18 Camille Roy, Manuel d'bistoire de la littérature canadienne, Québec, Imprimerie de l'Action sociale, 1918, X-120 p. 
- [l'abbé Jean-Baptiste-Antoine] Ferland fit de fortes études au collège de Nicolet. [... En conséquence] il comprit mieux que Garneau le caractère religieux de nos origines historiques.

Le manuel offre plusieurs exemples de ce type. Benjamin Sulte, Rémi Tremblay et Arthur Buies illustrent les problèmes que "rencontrent * les autodidactes et les mauvais sujets. Antoine Gérin-Lajoie, Joseph-Edmond Roy et Pamphile Lemay, pour ne citer que ceux-là, montrent la valeur de l'enseignement classique. ${ }^{19}$

C'est au Bulletin du parler français que la collaboration entre Camille Roy et Adjutor Rivard se fera la plus étroite. Issu de la Société du parler français au Canada, fondée en 1902 par l'abbé Stanislas Lortie, Eugène Rouillard et Adjutor Rivard, le Bulletin fut la pierre d'angle du régionalisme québécois. Dès ses premières livraisons, bien que l'orientation demeurât d'abord et principalement philologique - elle entendait veiller à la restauration et à la défense du "doux parler " des ancêtres - la revue fait une place importante à la littérature nationale en liant les deux destins. Lors du Premier grand congrès de la langue française au Canada en 1912, dont Adjutor Rivard avait été l'âme dirigeante, Camille Roy trace un premier bilan du travail de la Société en marquant de façon non équivoque son orientation régionaliste:

Déjà la Société du parler français au Canada avait quelque peu élargi, sans le briser, le cadre de ses premiers travaux. Elle avait sollicité pour son Bulletin des études d'histoire de la littérature canadienne, des articles de critique, des pages originales sur les mours et les choses de chez nous. Souvenez-vous seulement du Poêle de M. Adjutor Rivard et de son Heure des vaches. Et ditesmoi si des pages comme celles-là ne sont pas écrites dans une langue atel sur le papier qu'à la bouche», comme eût dit Montaigne, et si elles n'illustrent pas, comme il convient, le programme de notre Société ? ${ }^{20}$

19 Lucie Robert, Le Manuel d'histoire de la littérature canadienne de $M^{g^{r}}$ Camille Roy, Québec, Institut québécois de recherche sur la culture, coll. - Edmond-de-Nevers *, n 1, 1979, p. 116.

20 Camille Roy, la Société du parler français et son premier concours littéraire, Premier Congrès de la langue française au Canada. Compte rendu, Québec, Imprimerie de l'Action sociale, 1913, p. 339. 


\section{Une fortune scolaire}

Dans les années qui suivent, Camille Roy continue à faire la promotion de l'œuvre de Rivard. Dans ses Morceaux choisis, l'auteur du Manuel d'bistoire de la littérature canadienne présente deux textes de son collaborateur: "La maison", extrait de Chez nous, et "Les vieux mots", extrait des Études sur les parlers de France au Canada que Rivard avait publié en 1914. Pour le premier, il attire l'attention des maîtres et des élèves sur «l'art du vocabulaire et de la phrase * qui caractérise, selon lui, la manière de l'auteur et, pour le second, il les invite à répondre aux questions suivantes: "De quels éléments est formée notre langue? Quels sont les plus précieux, les plus capables d'assurer la continuité de son génie? "21

Grâce en particulier à Camille Roy qui l'accueillit dans son Manuel dès 1918, la fortune scolaire de Chez nous fut très importante. Fort de la consécration que lui accorde l'Académie française en juin 1920 avec le prix Davaine, le livre est offert en prix de fin d'année. Toutes les publications destinées aux enseignants en recommandent l'étude. On peut citer, à titre d'exemple, un article de l'abbé Émile Bégin, paru dans L'enseignement secondaire au Canada, qui propose une "lecture expliquée " du récit "Le poêle". Après s'être longuement attardé sur la définition des locutions canadiennes contenues dans ce récit que l'analyste qualifie de "croquis lyrique", Bégin s'adonne à un commentaire littéraire qui met en relief les détails de la composition et les subtilités du style. En conclusion, l'auteur insiste sur la valeur morale de l'œuvre:

[Cette litterature] est sincère et montre admirablement chez nos aïeux la vertu de respect qu'ils avaient pour tous les objets en rapports proches ou lointains avec leur existence. Ce respect menait tout droit à l'amour ou du moins à l'attachement attendri qui voit dans les choses à l'usage de l'homme une attention délicate du bon Dieu, une de ses innombrables bontés. ${ }^{22}$

Cette illustration de la réception scolaire de Cbez nous montre que l'auteur a accompli les buts qu'il s'était fixés, tant

21 Camille Roy, Morceaux choisis d'auteurs canadiens, Montréal, Librairie

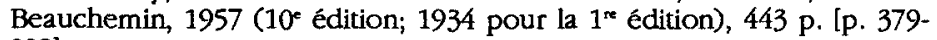
382].

22 Émile Bégin, *Versification. Lecture expliquée. "Le poêle ", L'enseignement secondaire au Canada, vol. XVIII, $\mathrm{n}^{\circ} 1$, octobre 1938, p. 78. (C'est l'auteur qui souligne). 
comme écrivain que comme philologue à la Société du parler français. Ses intentions étaient modestes. Et si, comme Camille Roy, il n'hésite pas à parler de "littérature canadienne", il situe sans hésiter les productions linguistiques et littéraires du Québec du côté des apports régionaux. *Essayons, écrit-il dans ses Études sur les parlers de France au Canada, de greffer sur le vieux tronc du français une tige canadienne, pleine de sève, saine, vigoureuse, et espérons aussi longtemps qu'il faudra. ${ }^{23}$. Pour eux, le diagnostic est clair: l'état de la langue française au Canada, marquée par l'éloignement de la Mère patrie et par la cohabitation avec l'anglais, langue inférieure en richesse et en maîtrise à celle qui est pratiquée par les écrivains de la Métropole, rend impossible le rêve d'une littérature autonome. C'est pourquoi, malgré les accents parfois autonomistes de leurs positions, les animateurs du régionalisme québécois conserveront toujours une attitude de respect admiratif à l'égard de la littérature française. En 1918, dans sa première livraison, L'action française, pourtant dévouée à la promotion d'une littérature nationale de type régionaliste, recommandait ce qui suit:

Voici les distributions des prix. L'occasion est excellente de faire de la bonne et fructueuse propagande. Que l'on voie à ne donner dans les écoles que des livres utiles, qui éveillent de nobles pensées et suscitent des sentiments généreux. Qu'à côté des chefs d'œuvre [sic] de la littérature française, on ait soin de donner et de multiplier les ouvrages du terroir, ceux qui apprendront aux enfants à mieux connaître, à aimer davantage leur pays. ${ }^{24}$

Les écrivains canadiens ne peuvent rivaliser sur le plan esthétique avec les écrivains français, mais ils peuvent et ont le devoir de témoigner des particularités de leur expérience en terre canadienne. Par là, ils participent à l'entreprise de *tous les poètes régionalistes " qui, selon Rivard, "pour aimer mieux la grande patrie, chérissent la petite; pour enrichir la langue, puisent à pleine plume dans les patois» ${ }^{25}$. Le programme est tracé: pour

23 Adjutor Rivard, "Les formes dialectales dans la littérature canadienne", Etudes sur les parlers de France au Canada, p. 86.

24 L'action française, vol. I, $n^{\circ} 1,1918$, p. 30. Cité par Pierre Hébert dans -Quand éditer, c'était agir. La Bibliothèque de L'Action française (19181927)., Reuse d'bistoire de l'Amérique française, vol. XIVI, no 2, automne 1992, p. 228.

25 A. Rivard, Etudes sur les parlers de France au Canada, p. 77. 
l'heure, il faut travailler à corriger et à raffermir le parler canadien, regénérer à ses sources classiques et paysannes cette «langue humaine" que les gens instruits et les gens des villes ne cessent de corrompre. Dans cette perspective, les écrivains canadiens ressemblent au poète illettré d'Adjutor Rivard; poètes naturels à qui manquent la formation et la discipline, ils ne peuvent aspirer qu'à devenir de "bons ouvriers ", de "vaillants travailleurs de la plume", selon les termes mêmes de l'abbé Camille Roy. Le recueil Chez nous illustre et réalise les potentialités de ce programme d'action mais il en dévoile aussi, dans sa thématique et ses structures, les limites et les ambiguités.

\section{Le murmure de la désolation}

À la lecture des douze proses brèves qui composent le recueil de Rivard, comme d'ailleurs à la lecture des innombrables petits récits paysans accueillis par la critique autorisée de l'époque comme des modèles de l'esthétique régionaliste et d'heureuses contributions à l'édification de la littérature nationale, s'impose une constellation de thèmes et d'images qui évoquent la disparition et la perte. Partout il est question d'objets oubliés, de lieux désertés, de pratiques révolues. Rivard décrit la maison abandonnée dont les enfants n'osaient s'approcher, le petit rosier "mort sans avoir donné sa fleur, sans avoir jeté son parfum", le vieux capitaine qui trépasse après avoir consenti à vendre sa vieille goélette qui était depuis longtemps déjà a embossée le long du quai vermoulu ", le feu qui détruit la grange neuve, les ravages du temps et du progrès qui menacent le ber ancestral, le vieux poêle et "la vraie charrette à foin ". Les objets et les êtres n'ont de réalité que celle que leur confère le souvenir. Ils appartiennent au passé, à un temps révolu ou en voie de l'être. Le présent n'existe pas, sinon comme lieu d'inscription de la mémoire d'un narrateurtémoin. L'histoire a cessé sa marche. Aucun horizon ne se dresse si ce n'est celui de la réminiscence et de la rumination éternelles. Les tableaux de Rivard ressemblent à des épitaphes dont les ans auraient effacé le détail des inscriptions. Il est étonnant de constater que ces récits qui se proposaient de célébrer une manière particulière d'être au monde, de marquer concrètement et symboliquement une identité et une appartenance, ne comportent aucune précision spatio-temporelle permettant de situer les lieux et les événements. La narration privilégie les génériques: la compagne, celle d'hier, celle de toujours jusqu'à cette cassure, jamais 
nommée, qui est venue mettre en péril la pérennité de ses traditions. L'espace et le temps ont perdu leur corps particulier pour entrer dans cet illo tempore dont parlait Mircea Eliade à propos du Mythe de l'éternel retour. Seul le lexique permet d'ancrer le récit dans un sol et une histoire. Par la mise en valeur des formes canadiennes qu'a pris chez nous le "doux parler des ancêtres", le récit singularise un propos qui pourrait être d'ailleurs, le rapatrie, l'habille de couleurs qui n'appartiennent qu'à lui:

Le plus souvent, c'était l'Histoire Sainte que Gédéon nous contait. Le récit du déluge, en particulier, était une merveille. Nous voyions se construire l'arche, une espèce de chaland avec une grange dessus, et dans laquelle il y avait, d'un bout à l'autre, des parés et des barrures, comme dans une écurie; puis le grand-père Noé, de sa bonne fourche, emplissait le fanil de foin, de beaucoup de foin à cause des éléphants qui mangeaient une botte par bouchée; ensuite, les animaux entraient, deux par deux, dans l'arche toute prête, et leur énumération était interminable. À la fin, la pluie se mettait à tomber, et Gédéon, qui avait vu l'inondation de la grande digue, devenait éloquent... Quand l'eau était rendue par-dessus les clochers d'églises, nous nous regardions épouvantés; j'en oubliais de mener la jument qui ne continuait pas moins, de son pas tranquille, à suivre la route tracée. ${ }^{26}$

L'extrait, comme d'ailleurs la majorité des récits du recueil, met en scène des enfants et des vieillards, des personnages totalement engagés dans l'univers paysan traditionnel, contrairement au narrateur qui, lui, possède des connaissances et un savoir qui lui permettent d'échapper à l'enlisement et à la destruction. Aussi peut-il se poser en guide pour le bon peuple:

Et dire qu'il y dans les villes, des gens qui n'ont jamais été en Grand'charrette! Les malheureux; ils ne savent rien de la vie! ${ }^{27}$

Pour le narrateur nostalgique, l'intérêt des pratiques anciennes reste lié à l'évocation des canadianismes ou des archaïsmes qui y sont rattachés. Leur union est si intime que la disparition de ces pratiques risque d'entraîner la disparition des mots qui servaient à les nommer et avec eux les signes convenus de l'identité canadienne. L'immobilité qui caractérise les petits univers clos et homogènes de Rivard semble la seule solution imaginée par le

26 Adjutor Rivard. *En grand'charrette., Chez nous, p. 52.

27 Ibid., p. 48. 
narrateur au problème que lui pose la marche du temps et du progrès:

Ne me parlez pas de votre charriot moderne [...] tel un squelette de guimbarde, une grotesque construction à claire-voie en forme de panier. $\mathrm{Ne}$ m'en parlez pas. ${ }^{28}$

Arrêter le temps. Arrêter l'histoire. Fixer les êtres et les choses dans la mémoire et le discours. Tourner le dos à ce qui vient. Tourner le dos à l'industrialisation qui est le fait des possédants Canadiens anglais, tourner le dos à l'urbanisation, à la réalité qui veut que déjà en 1901 un peu plus du tiers des Québécois habitent les villes et qu'en 1921 ils seront plus de la moitié. Refuser de voir la cassure, l'évoquer sans la nommer et faire comme la jument de la fable qui, indifférente au récit du Déluge, "n'en continuait pas moins, de son pas tranquille, à suivre la route tracée - et à n'entendre sans doute des bruits du monde et de la littérature que ces vieux mots du doux parler des ancêtres, "mots sans heurt et sans secousse 29 .

28 Ibid., p. 47.

29 Albert Lozeau, *Ie régionalisme littéraire. Opinions et théories *, Mémoires de la Sociếté royale du Canada, tome XIX, troisième série, 1921, p. 8 Ce texte est reproduit dans la section * Document * du présent numéro. 